\title{
Traumatic bronchial rupture: an unusual cause of tension pneumothorax
}

\author{
Shankar Hanamantrao Hippargi
}

Received: 11 September 2009 / Accepted: 27 December 2009/Published online: 9 April 2010

(C) Springer-Verlag London Ltd 2010

\begin{abstract}
Bronchial rupture is a rare and serious complication of blunt chest trauma, which can be easily overlooked on initial evaluation in the emergency room (ER) as the focus of the attending physician is diverted to more obvious external injuries which may not be of much significance. We present one such patient referred to us as a case of unresolved rightsided pneumothorax 2 days after intercostal drainage (ICD) tube placement.
\end{abstract}

Keywords Traumatic bronchial rupture $\cdot$ Tracheobronchial tear · Blunt airway injury

\section{Introduction}

Tracheobronchial tear refers to a mucosal tear or complete transection of the trachea or bronchi. Post-traumatic tracheobronchial tear is a rare, but life-threatening injury. It is a challenging diagnosis for the emergency physician and needs a high index of suspicion. Most of these injuries can be missed in the emergency room (ER) unless the physician suspects its possibility.

S. H. Hippargi $(\bowtie)$

Department of Accident \& Emergency Medicine, MMHRC, Lake Area, Melur Road,

Madurai, India

e-mail: drshank@rediffmail.com
Case

On 1 September 2009, a 25-year-old man was referred to our department as a case of road traffic accident (RTA) with right-sided tension pneumothorax with completely collapsed right lung. An intercostal chest drain (ICD) was inserted but there was no re-expansion of the right lung. The chest radiographs done before and after ICD insertion were the same.

Date and time of injury: 30 August 2009 at 11 p.m.

Mode of injury: patient was travelling in an auto, which overturned.

On arrival to our emergency department (ED) the patient was conscious and oriented, haemodynamically stable, but very dyspnoeic and his oxygen saturation was $80 \%$ on non-rebreathing mask. The patient's ICD on the right side was patent and functioning well. There was continuous and profuse bubbling noted in the ICD bag. There was extensive subcutaneous emphysema and absent breath sounds on the right side, with tracheal deviation to the left. A bedside chest radiograph was done immediately, which showed features of right-sided tension pneumothorax with completely collapsed right lung (Fig. 1). There was no change as compared with the previous chest radiographs.

When the ICD tube was connected to low-pressure suction the patient had symptomatic relief transiently. Hence, the possibility of bronchial injury was suspected and computed tomography (CT) of the chest was done, which showed right-sided tension pneumothorax, with ICD in situ, a large tear in the right main bronchus, multiple traumatic contusions of the left lung and multiple rib fractures (Fig. 2). Cardiothoracic surgeons' opinion was 


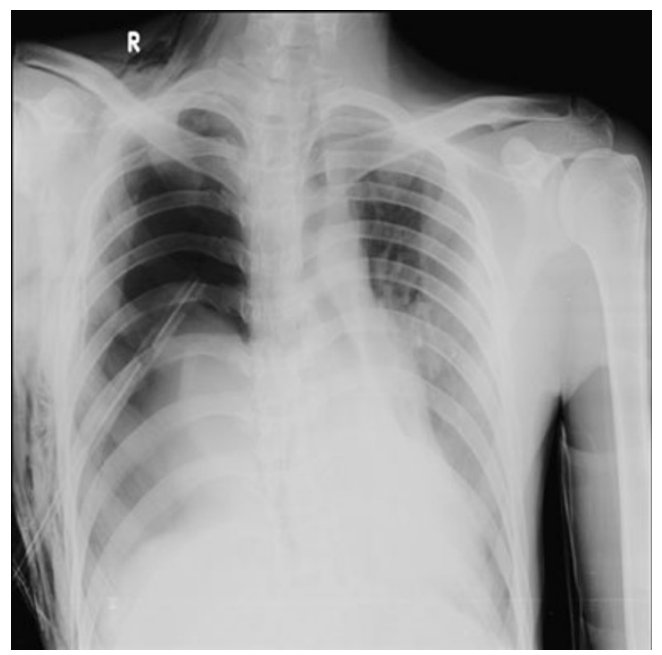

Fig. 1 Plain chest radiograph showing right-sided tension pneumothorax, collapsed right lung, ICD in situ, mediastinum and tracheal shift to left

sought and they advised immediate surgical repair with a possibility of right pneumonectomy as the bronchial defect was large. It was a case with high risk and poor prognosis, as the left lung too was contused extensively. The need for emergency surgery and prognosis were explained to the patient's relatives, but they were not willing to give consent for further management; hence, the patient was discharged against medical advice.

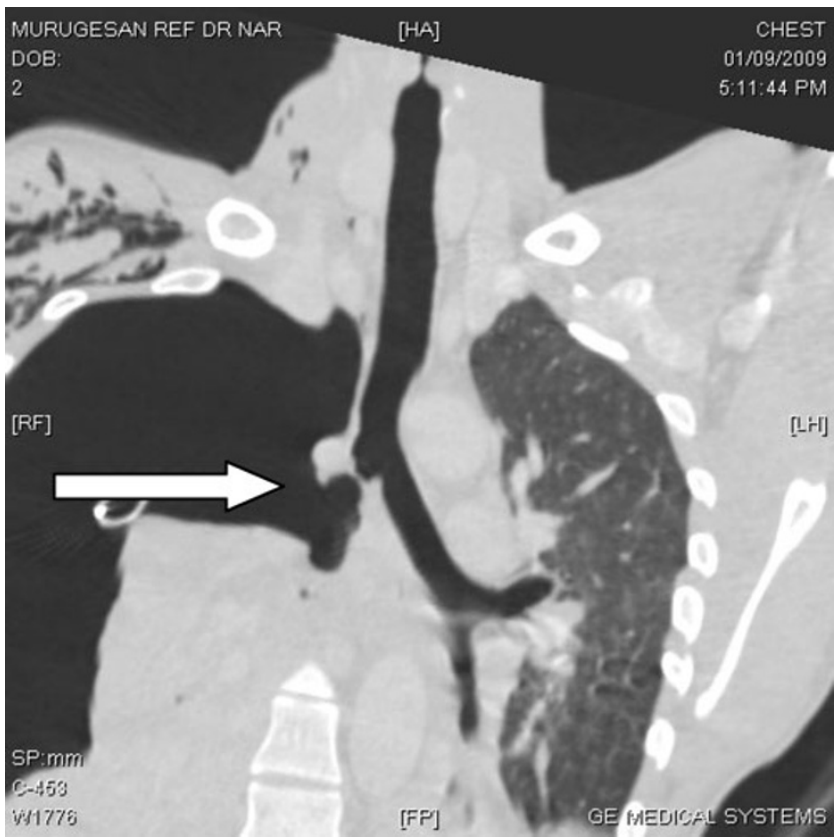

Fig. 2 Coronal section of $\mathrm{CT}$ of chest showing ruptured lateral wall of right main bronchus, collapsed lung and severe pneumothorax

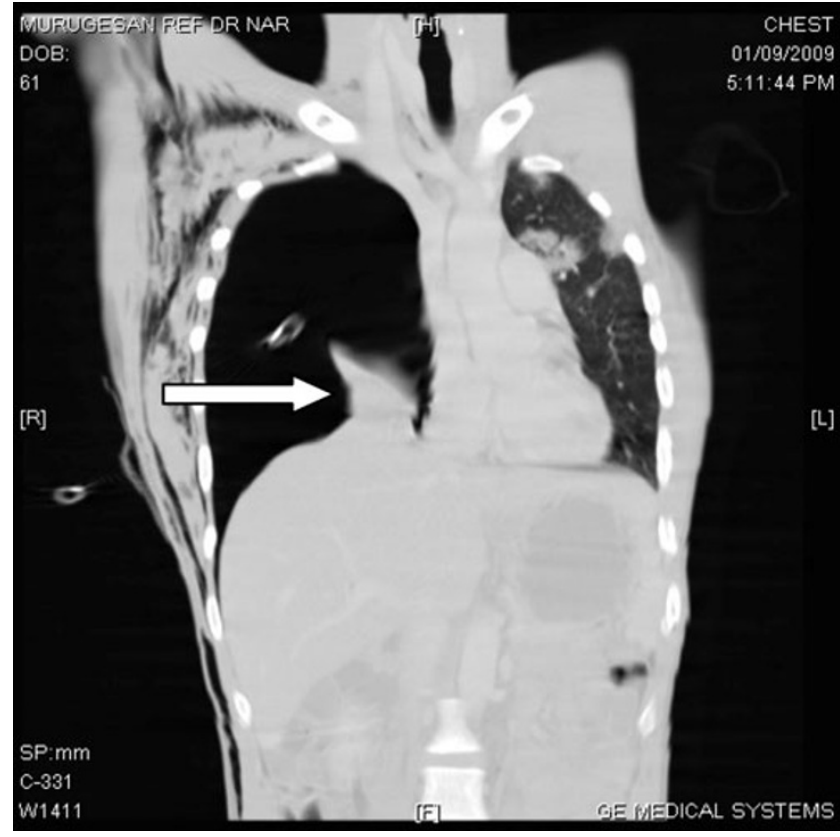

Fig. 3 Coronal section of CT of chest showing "fallen lung sign"

\section{Discussion}

A tracheal or bronchial rupture is a life-threatening lesion with non-specific presenting symptoms; hence, it is frequently unnoticed during the initial trauma assessment. The incidence of airway injury is about $1-3 \%$ of all blunt chest injuries [1]. Approximately $75 \%$ of the injuries occur within $2 \mathrm{~cm}$ from the carina; the right main bronchus is more commonly injured than the trachea or left main bronchus. Bronchial tear causing tension pneumothorax is an extremely rare case which can occur due to massive escape of air from a large bronchial tear as seen in this case. An unresolved pneumothorax after ICD insertion should immediately alert the treating physician to look for bronchial injuries.

\section{Signs and symptoms}

The signs and symptoms are non-specific which include dyspnoea, desaturation, hyper-resonance on percussion, decreased/absent breath sounds, hypotension, tracheal shift and distended neck veins. About $10 \%$ of the patients with tracheobronchial injury are asymptomatic [2]. Tracheal or bronchial injury should be suspected in patients with persistent or increasing pneumothorax, despite continuous intercostal drainage.

The best results in the treatment of such ruptures are obtained by the earliest possible restoration of the continuity of the air passages; hence, early diagnosis is vital for 
survival. Injuries to the trachea or proximal left main bronchus typically result in pneumomediastinum and not pneumothorax [1].

Possible mechanisms

The possible mechanisms for tracheobronchial injury are [2]:

- Injuries to bronchi occur due to rapid deceleration and shearing of mobile lung and bronchi from relatively fixed proximal structures.

- Forced expiration against a closed glottis causes a sudden rise in airway pressure and can cause a longitudinal tear in the bronchi.

- Compression between the sternum and vertebral column may cause bursting of these structures.

Diagnostic tests

The diagnosis is based on:

- The chest radiograph is the standard initial screening test in thoracic trauma. The radiographic manifestations of tracheobronchial injury are usually non-specific and include pneumomediastinum, pneumothorax, pneumoretroperitoneum, deep cervical emphysema and subcutaneous emphysema.

- The "fallen lung sign" is specific for complete bronchial transection. This sign describes the lung falling dependently, rather than collapsing centrally toward the hilum in the presence of a pneumothorax (Fig. 3).

- CT of the chest is the imaging modality of choice in tracheobronchial injuries; it not only confirms the diagnosis but also adds unique information. It has some limitations in detecting minor tears.

- Fibreoptic bronchoscopy is the best diagnostic tool for determining the location and extent of airway injuries
[3], and one should have a low threshold to perform it when the diagnosis is suspected.

Treatment

Laceration of the bronchus involving more than one third of the circumference needs surgical repair as it tends to cause severe stenosis with repeated lung infections and collapse [2]. Severe cases may require pneumonectomy. The prognosis after surgery depends on other associated injuries and the previous condition of the patient.

\section{Conclusions}

- Airway injury following blunt thoracic trauma is rare but can be life-threatening.

- Bronchial tear causing tension pneumothorax is very unusual.

- Early diagnosis in the ED is challenging as all the signs and symptoms are non-specific.

- A high index of suspicion is key for prompt diagnosis.

- The role of CT is vital in thoracic trauma.

Acknowledgement To the Department of Imaging Science, MMHRC, Madurai and the Department of Cardiothoracic \& Vascular Surgery, MMHRC, Madurai.

\section{References}

1. http://imaging.consult.com/chapter/S1933-0332(08)73224-5\# airway injury. Accessed 6 Sep 2009

2. Bowling WM, Wilson RF, Kelen GD, Buchman TG (2003) Thoracic trauma. In: Tintinalli JE, Kelen GD, Stapczynski S, Ma OJ, Cline DM (eds) Tintinalli's emergency medicine: a comprehensive study guide, 6th edn. McGraw-Hill Companies, Inc. New York, p 1675

3. http://priory.com/cmol/skobel.htm. Accessed 7 Sep 2009 